\title{
Especificação e Verificação Formal de um modelo de STI-PBL por Re- des de Petri Coloridas
}

\author{
Eliane Santiago Ramos \\ Faculdade Carlos Drummond de Andrade \\ Rua Prof. Pedreira de Freitas, 401/415 \\ CEP 03312-050 - São Paulo - SP \\ eliane.ramos@uol.com.br
}

\author{
José M. Parente de Oliveira \\ Divisão de Computação - Instituto Tecnológico de \\ Aeronáutica \\ Pça Marechal Eduardo Gomes, 50 \\ CEP 12228-900 - São José dos Campos - SP - Brasil \\ parente@ita.br
}

\begin{abstract}
Resumo Apresenta-se neste trabalho uma abordagem de modelagem por Redes de Petri Coloridas para especificação e verificação formal de um modelo de Sistema Tutor Inteligente que utiliza a Aprendizagem Baseada em Problemas como estratégia pedagógica. A especificação e a verificação formal permitem verificar se as funcionalidades planejadas do modelo pedagógico são realizadas, antes da etapa de implementação do sistema. Experimentos indicam consistência geral e beneficios da proposta.
\end{abstract}

Palavras-Chave: sistemas tutores inteligentes, aprendizagem baseada em problemas, Redes de Petri Coloridas.

\begin{abstract}
This research aims at presenting an approach of Color Petri Nets to formal specification and verification of an Intelligent Tutor System model that uses Problem-based Learning as a pedagogical strategy. The specification and verification formal allow to verifying whether the planned features of the pedagogical model are done before the system's implementing stage. Initial experiments indicate consistency and overall benefits of the proposal.
\end{abstract}

Keywords: intelligent tutoring systems, problem based learning, coloured petri nets.

\section{Introdução}

Sistemas Tutores Inteligentes (STIs) são sistemas de computação com propósitos educacionais, cujo objetivo é inferir o entendimento do aprendiz sobre o domínio a fim de adaptar, de maneira inteligente, a navegação através de materiais de aulas, leituras recomendadas ou seqüência de leituras apropriadas, conforme as necessidades individuais de cada aprendiz [1].

Normalmente, STIs são capazes de oferecer ajuda apenas no final de uma tarefa de solução de problema, quando comparam a solução apresentada pelo aprendiz com a solução representada no modelo especialista. O problema é que em processos de solução muito longos, o aprendiz pode permanecer em um caminho errado por muito tempo até que ele receba alguma orientação para seguir o caminho correto.

A avaliação dos passos intermediários no processo de solução pode ajudar o Sistema Tutor Inteligente (STI) a reconhecer a estratégia de solução planejada pelo aprendiz. Existem, na literatura, recomendações gerais sobre como organizar a avaliação destes passos [2]. Entretanto, a modelagem do processo de solução e o mecanismo de adaptação do aprendiz não são claros.

A definição da estratégia pedagógica na fase de requisitos de um STI se justifica, pois possui requisitos funcionais que impactam na especificação dos requisitos do sistema. É fato que erros na especificação de 
requisitos são os maiores responsáveis por grande parte do esforço e retrabalho em projetos de desenvolvimento de software. Isso acontece porque estes não são sempre documentados de um modo compreensivo e consistente; o escopo do projeto freqüentemente aumenta durante o desenvolvimento; quando os requisitos mudam, há pouca ou nenhuma análise do impacto das mudanças sobre o trabalho e sobre os objetivos do projeto.

O formalismo das Redes de Petri para representação dos passos e estados do sistema foi o fator de motivação para explorá-las. Além de ser uma teoria bem consolidada na literatura como um método formal de modelagem, verificação e simulação de sistemas.

O objetivo desta pesquisa é apresentar um modelo formal para especificação e verificação das funcionalidades de um projeto de STI, considerando o modelo pedagógico definido, a fim de apresentar um esquema operacional de uso, representar as interações disponíveis para o aprendiz de forma explícita, visando melhorar a visibilidade e a compreensão das funcionalidades do sistema, antes mesmo da fase de implementação.

Este artigo está organizado em oito seções, incluindo esta Introdução. A Seção 2 apresenta a fundamentação teórica e a explicação do contexto da pesquisa. A Seção 3 apresenta a caracterização do STI-PBL por meio de artefatos de modelagem. Na Seção 4 é apresentada a metodologia de especificação formal por Redes de Petri Coloridas. A Seção 5 a verificação formal dos aspectos funcionais e não funcionais do sistema. Na Seção 6 são apresentados a análise dos resultados obtidos na verificação do modelo. Na Seção 7 sintetizam-se os trabalhos relacionados e as principais contribuições a esta pesquisa. Por fim, as conclusões da pesquisa juntamente com as recomendações.

\section{Fundamentação Teórica e Contextualização da pesquisa}

Esta seção tem por objetivo apresentar os fundamentos teóricos para a consolidação do trabalho, que ajudarão na compreensão do contexto desta pesquisa e da abordagem de solução.

\subsection{O STI-PBL}

O conjunto de elementos que compõe a arquitetura de STIs é composto por quatro elementos com papéis bem definidos, sendo os Modelos do Domínio ou Especialista, Pedagógico, Aprendiz e Interface [3].

O Modelo do Domínio, também conhecido como Modelo Especialista é uma estrutura de dados que contém o conhecimento a ser ensinado ao aprendiz.
Contém O Modelo do Aprendiz é uma estrutura de dados que representa o estado de conhecimento do aprendiz sobre o domínio. O Modelo Pedagógico é responsável por decidir qual estratégia pedagógica utilizar para ensinar e como aplicá-la para alcançar os objetivos de aprendizagem.

O Modelo de Interface é o recurso que o aprendiz possui para interagir com o sistema.

Denomina-se nesta pesquisa STI-PBL o Sistema Tutor Inteligente que utiliza a Aprendizagem baseada em Problemas como modelo pedagógico.

A Aprendizagem baseada em Problemas (PBL, do inglês Problem based Learning) é uma estratégia pedagógica, centrada no aprendiz, na qual os processos de ensino e aprendizagem partem de problemas [4]. Como característica, é considerada uma abordagem construtivista, pois desafia o aprendiz a identificar seus saberes sobre o domínio e identificar quais habilidades e competências precisará desenvolver para resolver um determinado problema.

\subsection{Caracterização do Problema no STI-PBL}

O problema é um aspecto muito importante a ser considerado no STI-PBL, pois representa o meio pelo qual os objetivos da PBL serão alcançados.

Para aplicação da PBL, o problema deve ser a descrição de um cenário real e mal estruturado [5], cujo caminho para a solução não é conhecido pelo aprendiz [6], deve abranger alguns conceitos do domínio, e o processo de solução deve conduzir ao aprendizado de um número limitado de conceitos.

Para a especificação e verificação formal do STI-PBL, o problema deve ser compreendido, formalmente, como definido por Chang-Kai Hsu [7], ou seja, um estado de todas as suas descrições, a qual contém um conjunto de estados conhecidos $(\boldsymbol{\theta}) \mathrm{e}$ desconhecidos $\left(\boldsymbol{x}=\left\{\boldsymbol{x}_{\boldsymbol{i}}\right\}\right)$, denotado como $\boldsymbol{\psi}=\{\boldsymbol{\theta}, \boldsymbol{x}\}$. O processo de solução deve ser construído por alguns estados, a saber: $i)$ um estado final $\left(\boldsymbol{\psi}_{f}=\left\{\boldsymbol{\theta}_{f}\right\}\right)$, que corresponde ao estado objetivo, ou seja, sua solução; ii) um estado inicial $\left(\boldsymbol{\psi}_{0}=\boldsymbol{\theta}_{0}, \boldsymbol{x}_{\boldsymbol{0}}\right)$, que corresponde ao enunciado do problema; e iii) vários estados intermediá$\operatorname{rios}\left(\boldsymbol{\psi}_{I}=\boldsymbol{\theta}_{I}, \boldsymbol{x}_{I}\right)$, que descrevem os possíveis caminhos de solução.

O processo de solução de um problema é transformado de um estado $\boldsymbol{\psi}_{i}$ em outro estado $\boldsymbol{\psi}_{i+1}$ por meio de operadores de transição, os quais são denotados por $\delta_{j}\left(\boldsymbol{\psi}_{i}\right)=, \boldsymbol{\psi}_{i+1}$. A transição que muda o problema de um estado para outro pode ser representada como um arco entre dois estados. A estratégia de solução de problemas é $\delta_{j}=\xi_{k}\left(\left(\boldsymbol{\psi}_{i}, \boldsymbol{\psi}_{f}, \tau\right) \in \xi\right.$, onde $\tau$ corresponde ao conjunto de 
transições disponíveis no estado $\Psi_{i}$ para alcançar o estado final $\psi_{f}$.

Resumindo, um problema é composto por um enunciado $\left(\boldsymbol{\psi}_{0}\right)$, uma solução $\left(\boldsymbol{\psi}_{f}\right)$ e uma ou mais estratégia para alcançar a solução $(\delta)$.

Este trabalho não trata heurísticas para reduzir a distância entre os estados inicial e final, mas utiliza o formalismo para a identificação do estado mais apropriado para oferecer ajuda e avaliação nos passos intermediários de processos de solução de problemas.

\subsection{Aplicação da PBL no STI-PBL}

Em STIs, a definição e o detalhamento da estratégia pedagógica é um aspecto importante, porque a implementação da estratégia implica diretamente na implementação das funcionalidades do sistema [8].

Partindo da organização do problema apresentada na seção 2.2, a Figura 2.1 representa um esquema aplicado para a PBL, o qual foi definido originalmente por Oliveira et al. [9]. Este esquema foi elaborado a partir dos princípios definidos em Labidi e Ferreira [10,11], nos princípios de projetos organizados por solução de problemas definidos em Barges [12] e no esquema apresentado em Savery e Duffy [13], para uso em estudo individual.

O esquema segue um fluxo com seis fases e o conjunto de todas as fases constitui uma seção de estudos. $\mathrm{Na}$ fase Preparação do Aprendiz é feita uma apresentação dos objetivos do curso e da metodologia de ensino. Depois, uma enquete sobre o domínio é aplicada para identificar o conhecimento, as preferências e os estilos cognitivos do aprendiz, definindo assim a primeira modelagem do aprendiz.

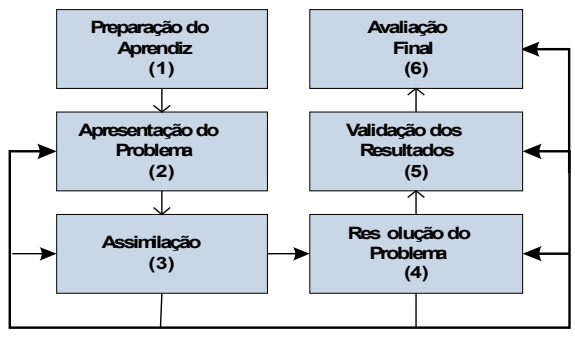

Figura 2.1. Esquema aplicado para a PBL [14]

$\mathrm{Na}$ próxima fase um problema é apresentado, de acordo com o nível de conhecimento do aprendiz. Esta fase é denominada Apresentação do Problema.

A Assimilação é a fase que permite ao aprendiz fazer esclarecimentos sobre o domínio, pesquisar os conceitos abordados no problema, buscar o significado dos termos desconhecidos e, baseado no conhecimento empírico ou científico que possui, construir suas hipóteses de como resolverá o problema.

A definição das hipóteses é uma forma de representar, explicitamente, as várias linhas de raciocínio que o aprendiz construiu sobre o problema. Entretanto, após investigar os assuntos considerados relevantes para cada hipótese, através do estudo individual, o aprendiz pode eliminar algumas hipóteses e estabelecer fatos. Fatos são conclusões do aprendiz dos conceitos que ele aplicará para resolver o problema. Ao final, o aprendiz deve estabelecer um plano de ação.

Na fase Resolução de Problemas, o plano de ação estabelecido na fase anterior é executado. O aprendiz se auto-avalia por meio da revisão das hipóteses, aplicação de novos conhecimentos e reformulação do plano de ação estabelecido na fase anterior.

Na fase Validação dos Resultados, a solução do aprendiz é comparada com a solução do modelo especialista. Caso a solução do aprendiz seja reconhecida pelo Modelo Especialista como correta, o aprendiz é redirecionado para a etapa de Apresentação do Problema, onde problemas abrangendo outras unidades de aprendizagem serão apresentados. Entretanto, caso o aprendiz não tenha sucesso na solução do problema atual, ele é redirecionado à fase de assimilação, a fim de explorar os conteúdos instrucionais e investigar os conceitos relevantes à solução do problema.

A última fase do processo é a Avaliação Final. Nesta fase, o aprendiz é submetido a uma avaliação que engloba um ou mais problemas cobrindo todas as unidades de aprendizagem. O objetivo é avaliar se o aprendiz é capaz de resolver problemas e de transferir habilidades de solução entre problemas estruturalmente similares.

\subsection{Redes de Petri Coloridas para simulação}

As Redes de Petri Coloridas (CPN, do inglês Coloured Petri Nets) são uma ferramenta gráfica e matemática, úteis para modelagem formal de sistemas de software. Como características, podem ser executadas, são capazes de representar modelos com alto nível de abstração, além de fornecerem uma estrutura para projeto, especificação, verificação e validação de sistemas [15].

Possuem uma representação gráfica que contém os mesmos elementos básicos das Redes de Petri [16,17]. Adicionalmente, cores são atribuídas às marcas e cada cor pode ser comparada a um tipo abstrato de dado, conforme ilustrado na Figura 2.2. 


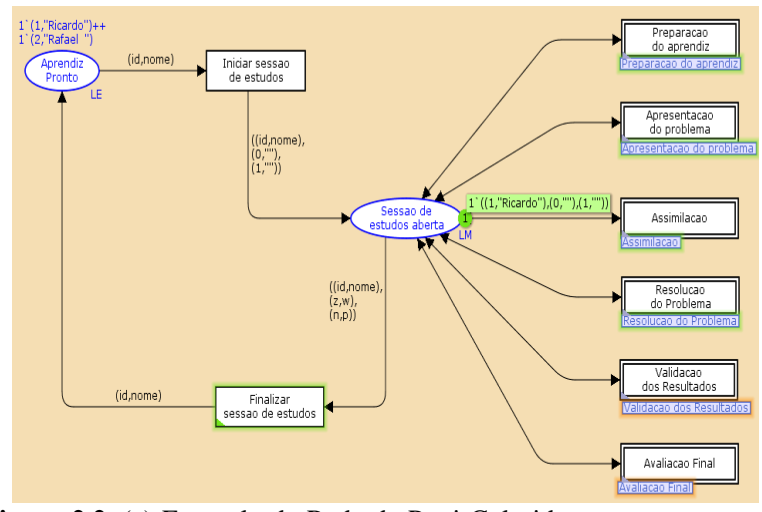

Figura 2.2. (a) Exemplo de Rede de Petri Colorida para o processo de autenticação do aprendiz (Adaptada de [18])

Os círculos representam estados do sistema e são definidos formalmente como $\mathrm{P}=\left\{p_{0}, p_{1}, \ldots, p_{n}\right\}$, um conjunto finito de lugares (places). Os lugares podem ser exemplificados como unidades pedagógicas dentro de um contexto instrucional.

As barras representam transições ou eventos. $\mathrm{O}$ conjunto $\mathbf{T}=\left\{t_{0}, t_{1}, \ldots, t_{n}\right\}$ pode representar unidades de

interação do usuário com o sistema, regras para mudança de estados, âncoras de navegação que, quando disparadas, provocam uma mudança comportamental no sistema, fazendo com que o estado do sistema mude.

Os textos acima dos círculos são marcas e indicam as informações presentes em cada lugar. O número de marcas em cada lugar indica o estado atual da rede e incorpora uma semântica que representa o estado do sistema antes e depois de cada evento. A notação $\mathbf{M}_{0}=\mathrm{P} \rightarrow\{2,0\}$ é uma marcação inicial, indica que há duas marcas no lugar $p_{1}$ e nenhuma marca no lugar $p_{2}$, representando assim o estado inicial do sistema. $\mathrm{O}$ exemplo da Figura 2.2 representa a marcação após o disparo da transição Iniciar sessão de estudos.

Os arcos definem relações de causalidade $(\mathrm{F})$ entre eventos e modificação do estado do sistema. Os lugares ligados a transições (PxT) são lugares de entrada e (T $x \mathrm{P})$ são lugares de saída. Um lugar pode ser de entrada para uma transição e de saída para outra, como pode ser observado na Figura 2.2, onde o lugar Aprendiz pronto é entrada para a transição Iniciar sessão de estudos e o lugar Sessão de estudos aberta é lugar de saída.

Formalmente, T é o conjunto de arcos usados para especificar como se dá a transformação de um estado em outro pela ocorrência de eventos no sistema.

Uma transição é dita estar habilitada se para cada lugar de entrada $\boldsymbol{p}$ para a transição $\boldsymbol{t}$ contém um número de marcas igual ao peso do arco que conecta $\boldsymbol{p}$ a $\boldsymbol{t}$. $\mathrm{O}$ disparo da transição $\boldsymbol{t}$ remove de cada lugar de entrada $\boldsymbol{p}$ o número de marcas correspondente ao peso do arco orientado que conecta $\boldsymbol{p}$ a $\boldsymbol{t}$ e coloca nos lugares de saída o número de marcas correspondente ao peso do arco.

Um lugar na CPN possui as propriedades cor, identificação e marcação inicial. A cor especifica o tipo de marca que o lugar poderá guardar. Três propriedades classificam as RdP como Coloridas:

a) marcações: possuem uma semântica específica no contexto do sistema, podendo representar, dentre outras coisas, recursos ou estados do sistema.

b) declarações: são notações usadas para declarar cores (ou tipos), funções, valores constantes e variáveis que são usadas nas inscrições. As declarações traduzem as especificações do sistema para uma linguagem reconhecida no modelo.

c) inscrições: podem ser de arco, transição ou de lugar. As inscrições de lugar especificam o tipo de marca que o lugar poderá guardar e definem as marcações iniciais. As de transição são cláusulas de guarda, as quais estabelecem a regra para o disparo da transição. As de arco especificam expressões ou regras, as quais definem o peso dos arcos.

A execução de modelos descritos em CPN implica na possibilidade de investigar o comportamento do sistema por simulações do modelo.

\section{Caracterização de um STI-PBL}

A construção de STI-PBL enfrenta, dentre outros, dois grandes desafios. $\mathrm{O}$ primeiro refere-se à falta de um padrão que permita o reconhecimento de uma atividade de aprendizagem como a PBL. O segundo, comum a outros tipos de ambientes de apoio à aprendizagem, refere-se a como modelar e representar a interação do aprendiz com o ambiente.

Embora o reconhecimento da importância da proposta pedagógica não seja novidade, a modelagem da interação do aprendiz com o STI dirigida pela proposta pedagógica, com o propósito de apoiar a modelagem e implementação do próprio STI, ainda é um caminho a ser explorado.

Com foco no segundo desafio, o objetivo da caracterização de um STI-PBL é apresentar uma visão geral do sistema e um cenário funcional por meio de um modelo de estados e interação, a fim de estabelecer um padrão operacional para o modelo pedagógico escolhido, representado na Figura 2.1.

Com o objetivo de fazer a especificação do sistema, caracterizou-se o STI-PBL por meio de três artefatos: i) a descrição da arquitetura; ii) visão dos casos de uso; e 
iii) modelagem de cenários funcionais por meio de Diagramas de Estados e Interação.

\subsection{Arquitetura do STI-PBL}

Um STI-PBL, no contexto deste trabalho, contempla os elementos da arquitetura clássica apresentados na Seção 2.1 e mais 5 elementos adicionais, propostos por Jacinto e Oliveira em [19], apresentados na Figura 3.1.

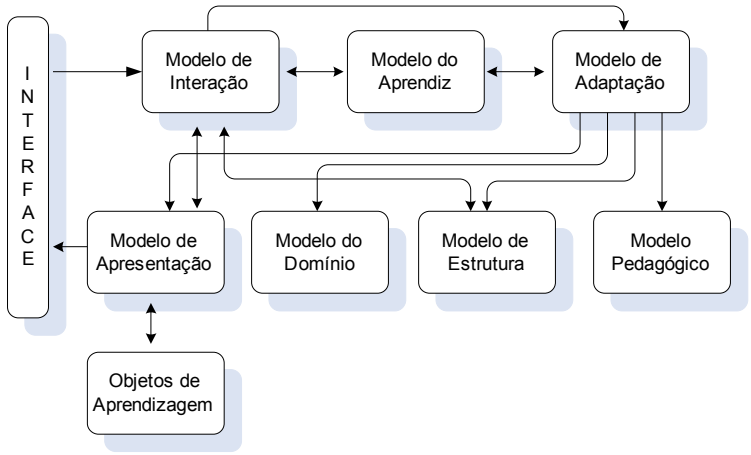

Figura 3.1. Arquitetura do STI-PBL [19]

$\mathrm{O}$ modelo de interação representa a descrição dos possíveis comportamentos do aprendiz. O modelo de estrutura especifica como os conceitos do domínio estão agrupados dentro de unidades semânticas. O modelo de adaptação contém uma base de regras, axiomas, e heurísticas para a tomada de decisão. O modelo de apresentação é responsável por compor a informação a ser apresentada ao aprendiz com base nas decisões do modelo de adaptação.

Nesta arquitetura, o modelo do domínio é capaz de atuar como modelo caixa de vidro [1,14] conforme ilustrado na Figura 3.2.

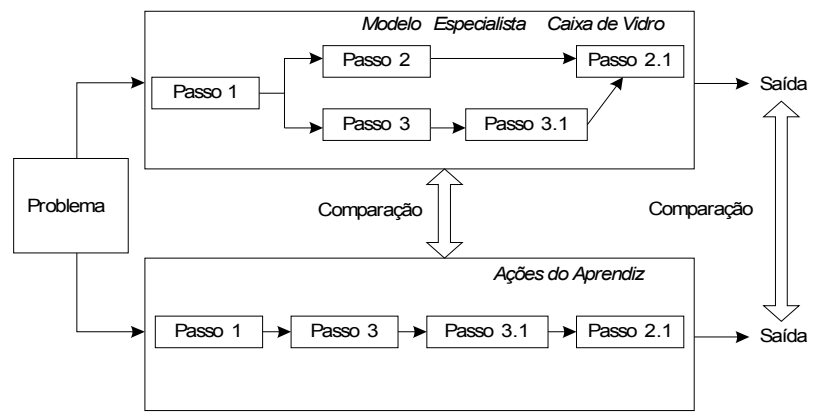

Figura 3.2. Representação do Modelo Especialista Caixa de Vidro

$\mathrm{Na}$ arquitetura clássica, o modelo pedagógico tem a responsabilidade de decidir como ensinar. Entretanto, com a separação de interesses entre os elementos da arquitetura da Figura 3.1, o STI-PBL é capaz de comparar as ações do aprendiz com as ações conhecidas no Modelo Especialista e, por meio do Modelo de Adaptação, inferir se o caminho que o aprendiz está traçando o levará ou não a uma solução.

\subsection{Especificação Formal dos Requisitos}

A partir da definição da arquitetura, descreve-se a visão geral dos requisitos do STI-PBL. Como a especificação formal dos requisitos não é simples de ser compreendida, a fim de tornar o processo mais simples, o

documento de requisitos é apresentado em duas etapas: i) visão dos casos de uso; e ii) cenário funcional por diagramas de estados e interação.

Os casos de uso da etapa Assimilação foram definidos a partir da definição da estrutura para aplicação da fase, demonstrado na Figura 3.3.

\begin{tabular}{|c|c|c|c|}
\hline \multicolumn{4}{|c|}{$\begin{array}{c}\text { ASSIMILAÇÃO } \\
\text { A PARTIR DA APRESENTAÇÃO DO PROBLEMA }\end{array}$} \\
\hline \multicolumn{4}{|c|}{$\begin{array}{l}\text { 1. Leitura do Problema } \\
\text { 2. O modelo especialista descreve o desempenho } \\
\text { requerido e as tarefas necessárias para resolver o problema } \\
\text { 3. O modelo do aprendiz deve representar as tare- } \\
\text { fas para resolver o problema }\end{array}$} \\
\hline $\begin{array}{l}\text { HIPÓTESES } \\
\text { (idéias) }\end{array}$ & FATOS & $\begin{array}{l}\text { ASSUNTOS DE APREN- } \\
\text { DIZAGEM }\end{array}$ & PLANO DE AÇÃO \\
\hline $\begin{array}{c}\text { Conjecturas } \\
\text { do aprendiz } \\
\text { com respeito } \\
\text { ao problema } \\
\text { apresentado. } \\
\text { Pode envol- } \\
\text { ver causas, } \\
\text { efeitos, con- } \\
\text { ceitos relaci- } \\
\text { onados ao } \\
\text { processo de } \\
\text { solução do } \\
\text { problema, } \\
\text { etc. }\end{array}$ & $\begin{array}{c}\text { Um resumo } \\
\text { das informa- } \\
\text { ções obtidas } \\
\text { através da in- } \\
\text { vestigação, } \\
\text { importante } \\
\text { para a gera- } \\
\text { ção da hipó- } \\
\text { tese }\end{array}$ & $\begin{array}{c}\text { Conteúdos que o } \\
\text { aprendiz precisa } \\
\text { conhecer ou en- } \\
\text { tender a fim de } \\
\text { completar a tarefa } \\
\text { de solução do pro- } \\
\text { blema }\end{array}$ & $\begin{array}{c}\text { Ações e seqüen- } \\
\text { ciamento das } \\
\text { ações que preci- } \\
\text { sam ser realiza- } \\
\text { das a fim de rea- } \\
\text { lizar a tarefa de } \\
\text { solução do pro- } \\
\text { blema }\end{array}$ \\
\hline
\end{tabular}

4. Raciocinando através do problema

O que o aprendiz faz com as colunas no quadro

\begin{tabular}{|c|c|c|c|}
\hline $\begin{array}{c}\text { HIPÓTESES } \\
\text { (idéias) }\end{array}$ & FATOS & $\begin{array}{c}\text { ASSUNTOS DE APRENDI- } \\
\text { ZAGEM }\end{array}$ & PLANO DE AÇÃo \\
\hline $\begin{array}{c}\text { Expansão/ } \\
\text { Foco }\end{array}$ & $\begin{array}{c}\text { Sintetiza e } \\
\text { Re-Sintetiza }\end{array}$ & Identificação & $\begin{array}{c}\text { Formularo } \\
\text { plano }\end{array}$ \\
\hline
\end{tabular}

5. O modelo especialista é capaz de apresentar retorno e avaliação para o aprendiz sobre suas escolhas em cada uma das etapas.

Figura 3.3. Esquema para a etapa Assimilação (Adaptado de [13]) 


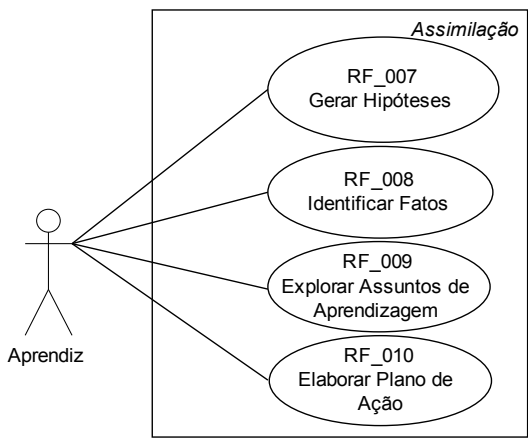

(a)

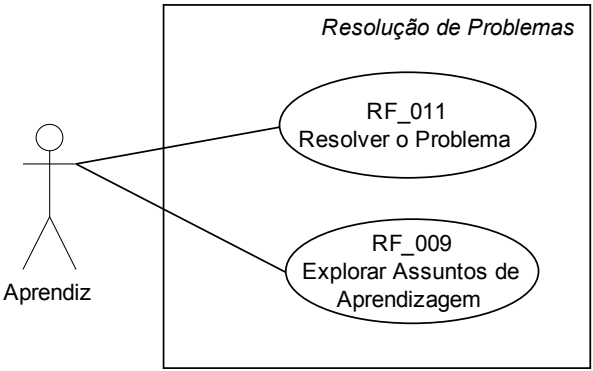

(b)

Figura 3.4. Visão dos Casos de Uso. (a) Etapa Assimilação. (b) Fase Resolução de Problemas

O esquema da Figura 3.3 descreve o processo da fase Assimilação do esquema proposto para a PBL. Adicionalmente, oferece um processo coerente para que o aprendiz possa refletir sobre as variáveis do problema, investigar os conceitos e planejar uma sequência para aplicá-los (plano de ação). A Figura 3.5 mostra o esquema para o processo de Resolução de Problemas.

\section{RETORNO E AVALIAÇÃO NO}

PROCESSO DE SOLUÇÃO DO PROBLEMA

1. Recursos utilizados e autocrítica

2. Reavaliar o problema e reformular suas deci-

sões.

\begin{tabular}{|c|c|c|c|}
\hline $\begin{array}{c}\text { IDÉlAS } \\
\text { (Hipóteses) }\end{array}$ & FATOS & $\begin{array}{c}\text { ASSUNTOS DE } \\
\text { APRENDIZAGEM }\end{array}$ & $\begin{array}{c}\text { PLANO DE } \\
\text { AÇÃO }\end{array}$ \\
\hline Revisão & $\begin{array}{c}\text { Aplicar novos } \\
\text { conhecimentos } \\
\text { e re-sintetizar }\end{array}$ & Identificar novos & $\begin{array}{c}\text { Re-formu- } \\
\text { lar deci- } \\
\text { (se necessário) }\end{array}$ \\
\hline
\end{tabular}

\section{APRESENTAÇÃO DO DESEMPENHO}

\section{CONCLUSÃO FINAL DO PROBLEMA}

1. Abstração do Conhecimento e sumário (desenvolver definições, diagramas, listas, conceitos, abstrações, princípios)

2. auto-desenvolvimento

- raciocínio através do problema;

- desbravando sem informação;

- auto-contribuições com suas tarefas

- aquisição ou refinamento do conhecimento.

Figura 3.5. Esquema para a etapa Resolução de Problemas (Adaptado de [13])

De acordo com o esquema apresentado em Savery e Duffy [13], seis funcionalidades básicas são identificadas, sendo quatro apresentadas na fase Assimilação e duas na fase Resolução do Problema. A Figura 3.4 mostra a visão dos casos de uso e apresenta-se uma breve narrativa. a) Gerar hipóteses: propicia ao aprendiz a possibilidade de identificar as possíveis abordagens de solução para o problema apresentado, representar suas idéias sobre as origens do problema e da estratégia de solução. Por outro lado, propicia ao STI a visibilidade dos aspectos cognitivos do aprendiz, ou seja, as crenças que o aprendiz possui sobre o caminho de solução. O objetivo é estimular o aprendiz a fazer uma leitura crítica do problema a fim de estruturá-lo.

b) Identificar fatos: propicia ao aprendiz a possibilidade de eliminar hipóteses e representar os conhecimentos que serão aplicados no processo de solução do problema.

c) Explorar assuntos de aprendizagem: constitui o ponto central do processo de assimilação e estruturação do problema. Essa funcionalidade propicia ao aprendiz a possibilidade de ler conteúdos de aprendizagem, ver diferentes objetos de aprendizagem e buscar esclarecimentos sobre os conceitos desconhecidos.

d) Elaborar plano de ação: tem por objetivo propiciar ao aprendiz a possibilidade de descrever os passos para a solução do problema, ou seja, sua estratégia de solução.

e) Resolver o problema: assistente que executa as instruções mapeadas no plano de ação, na ordem que foi estabelecida pelo aprendiz.

f) Explorar Assuntos de Aprendizagem: essa funcionalidade permite ao aprendiz voltar à etapa de Assimilação e torna todas as atividades de interação dessa etapa disponíveis para uso. O objetivo é investigar, refinar suas hipóteses, fatos ou até mesmo, redefinir o plano de ação estabelecido.

Com base na visão dos casos de uso, um cenário funcional em diagramas de estados e interação foi construído, o qual mostra os percursos possíveis do 
aprendiz pelo sistema e as atividades de interação disponíveis em cada estado. Dessa forma, os estados pro-

\subsection{Cenários funcionais por meio de diagramas de estados e interação}

Denominam-se, nesta pesquisa, atividades de interação todos os eventos que dependam ou não da ação do aprendiz para ocorrer. A cada interação, o sistema muda de estado e as atividades de interação podem mudar ou permanecer.

Os diagramas de estados de interação aqui apresentados são extensões dos Diagramas de Estados de Navegação [20], com foco não apenas na modelagem da interface, mas também na representação do modelo de interação da arquitetura para STI-PBL apresentada na Figura 3.1. piciam uma visão do funcionamento do tutor.

O modelo de interação representa o fluxo dentre as atividades de interação do STI-PBL e também uma visão do funcionamento do tutor, incluindo os objetos da interface a serem implementados.

$\mathrm{Na}$ atividade de Apresentação do Curso, representada na Figura 3.6, são apresentados os objetivos, as competências e habilidades a serem desenvolvidos. Os principais conceitos do modelo do domínio são representados por mapas conceituais [21].

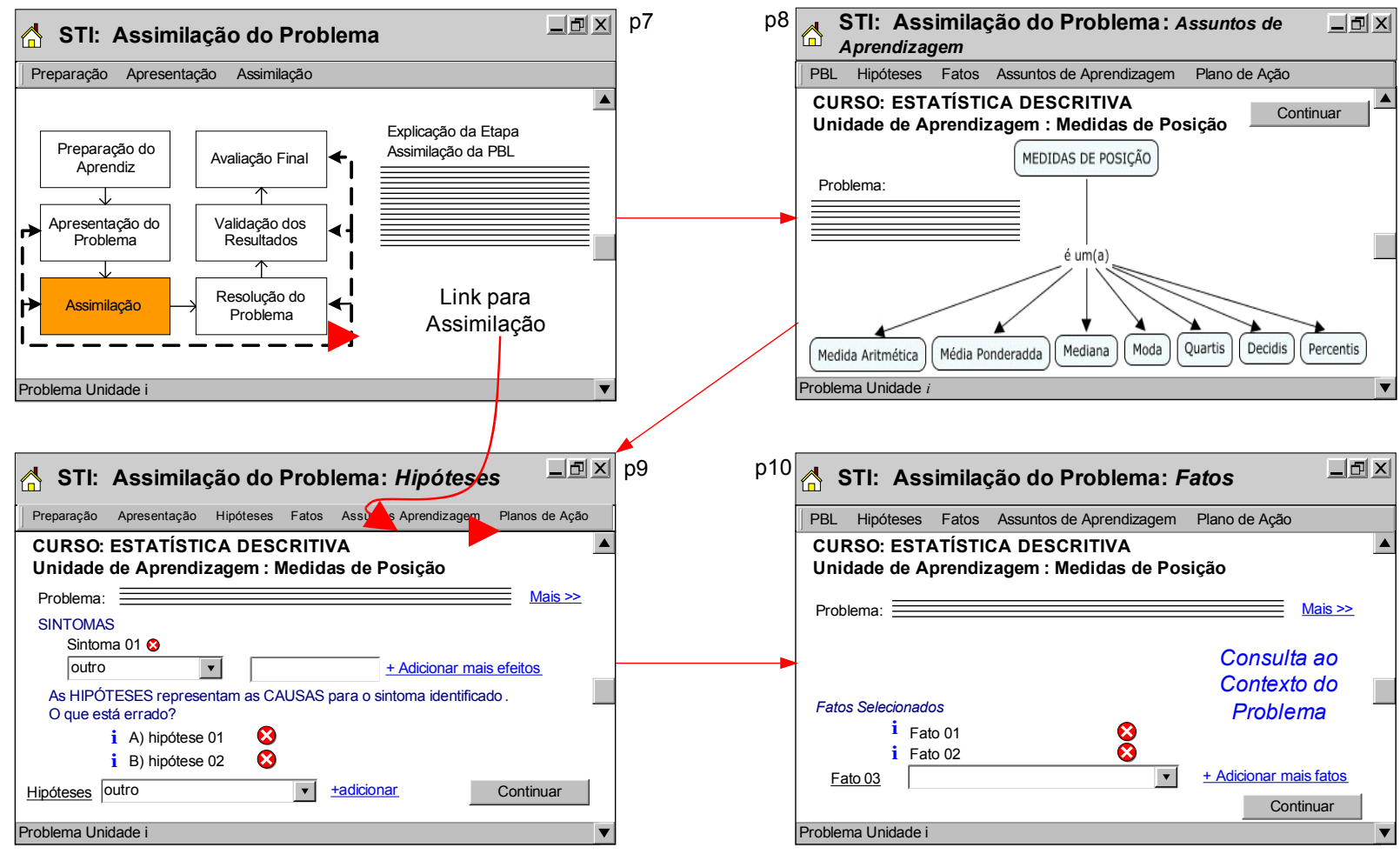

Figura 3.6. Diagramas de Estados e Interação da fase Assimilação 


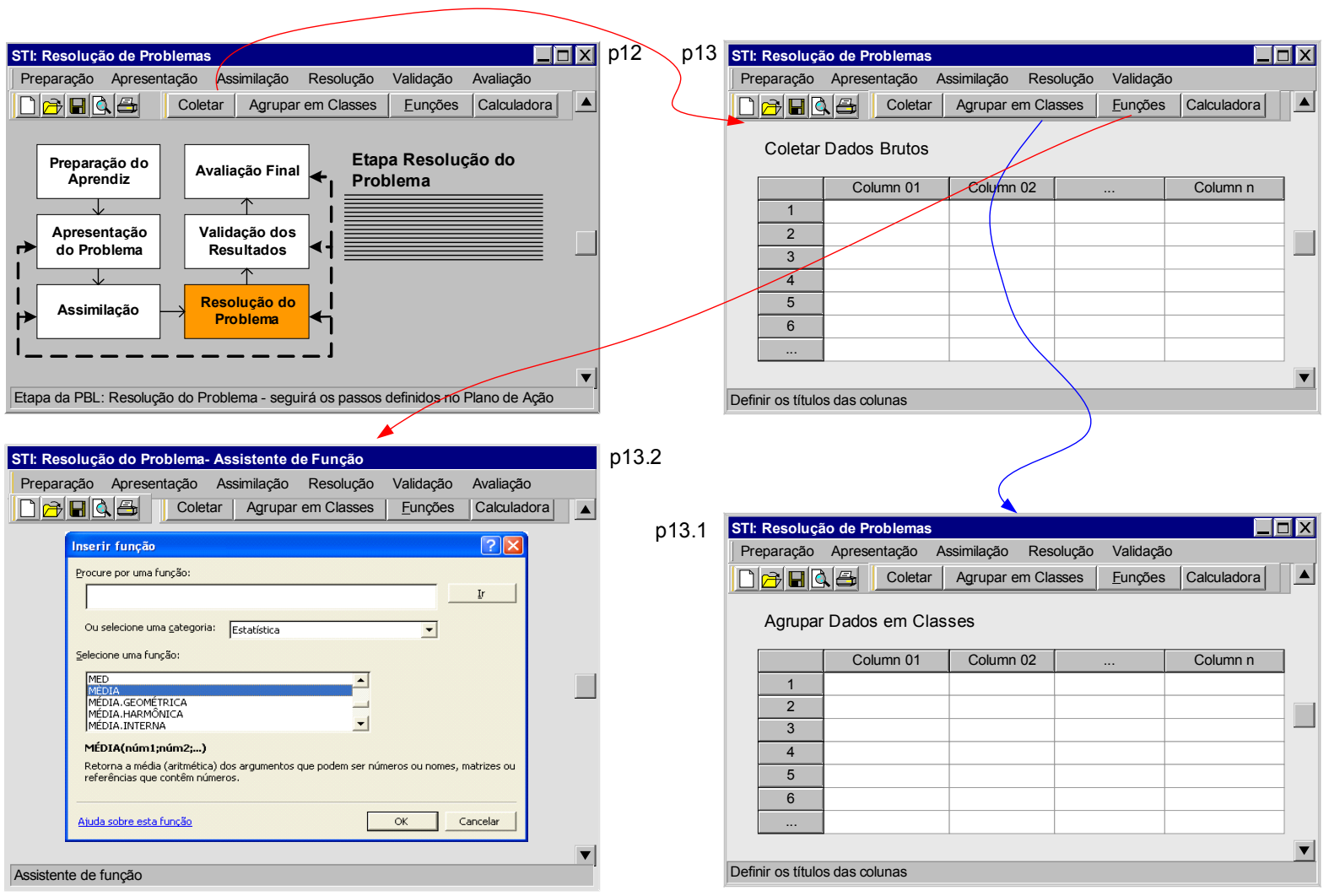

Figura 3.7. Diagramas de Estados e Interação da fase Resolução de Problemas

\section{Modelo do STI-PBL por Redes de Petri Coloridas}

O modelo CPN integra a arquitetura para STI-PBL apresentada na Figura 3.1 com o esquema aplicado para a PBL da Figura 2.1. As atividades de modelagem são apoiadas pela ferramenta CPN Tools [22], a qual fornece um ambiente gráfico para edição, verificação e análise dos aspectos comportamentais das redes.

A apresentação do modelo por CPN segue a mesma ordem usada para introduzir o esquema aplicado para a PBL. Considera-se, neste trabalho, CPN como linguagem de modelagem.

A modelagem do sistema é uma etapa que consiste em construir um modelo do sistema e derivar dele todos os comportamentos possíveis, enquanto a especificação consiste em especificar os comportamentos desejáveis do sistema [23].

A partir da caracterização do STI-PBL, estabeleceu-se uma correspondência funcional entres os estados de interação apresentados no cenário funcional e as Redes de Petri. Os lugares e transições correspondem, respectivamente, aos estados do sistema e os eventos disponíveis em cada estado, conforme pode ser observado na Figura
4.1.

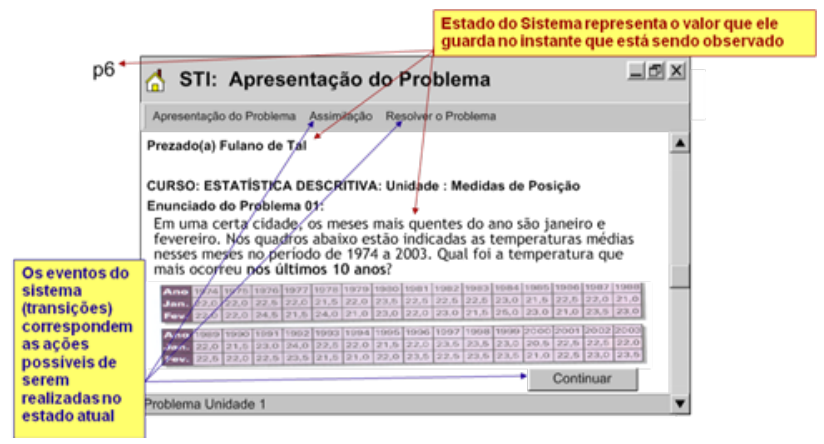

Figura 4.1. Um estado do sistema e os eventos disponíveis neste estado

Muito embora a PBL seja aplicada a grupos de estudos, o esquema adotado na Seção 2.3 tem foco no estudo individual. O modelo a seguir representa uma sessão de estudos iniciada pelo aprendiz no STI-PBL. O objetivo é verificar se os requisitos funcionais especificados para o aprendiz estão contemplados no modelo.

O primeiro nível da rede, demonstrado na Figura 4.2, foi graficamente organizado de modo a representar as etapas do esquema adotado para a PBL, e representa uma sessão de estudos iniciada pelo aprediz no STI-PBL.

O modelo é uma Rede de Petri Colorida e Hierárquica 
cujas marcas correspondem a instâncias do modelo do aprendiz, as transições correspondem a ações que cada aprendiz pode realizar e as transições com bordas duplas são transições de substituição, que encapsulam os processos das etapas da PBL.

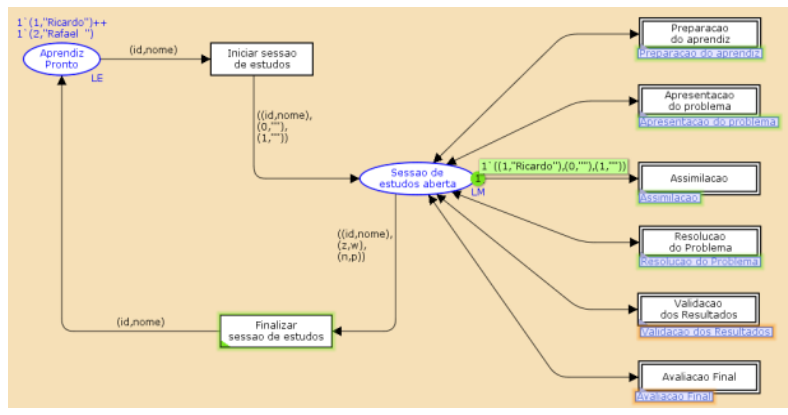

Figura 4.2. Hierarquia do modelo da sessão de estudos de um aprendiz

A Figura 4.2 mostra que uma sessão de estudos é iniciada quando um aprendiz solicita a sua entrada no sistema através do processo de autenticação (fornecimento de login e senha), e permanece viva até a saída do mesmo pelo disparo da transição Finalizar sessão de estudos. Uma vez autenticado, o aprendiz estará apto a fazer um conjunto de interações com o STI-PBL, respeitando as etapas da PBL e os pré-requisitos para mudança entre as etapas. Apresenta-se a seguir os modelos para cada etapa do esquema da PBL da Figura 2.2.

\section{Preparação do Aprendiz}

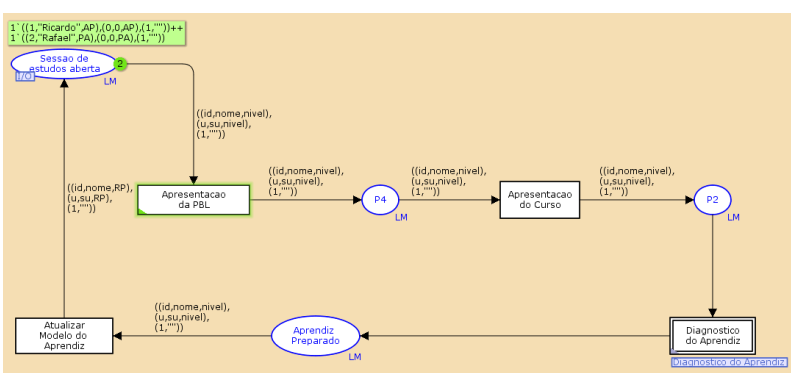

Figura 4.3. Modelo CPN da etapa Preparação do Aprendiz

$\mathrm{Na}$ etapa inicial do processo de aprendizagem, ao aprendiz é disponibilizado algumas interações, tais como apresentação do curso e da estratégia pedagógica PBL, bem como fazer o diagnóstico do aprendiz. No modelo da Figura 4.3, o diagnóstico do aprendiz é uma transição de substituição explorada na Figura 4.4.

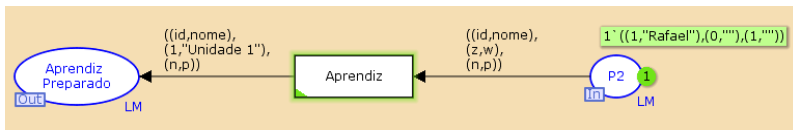

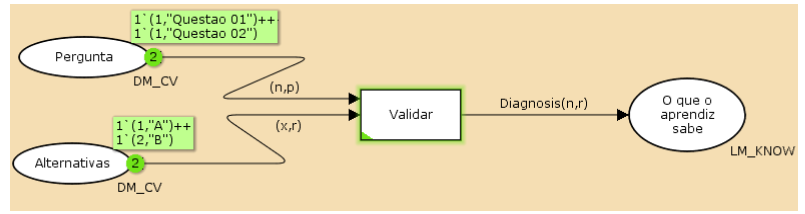

Figura 4.4. Processo de Diagnóstico do Aprendiz, responsável pela primeira modelagem do aprendiz.

O processo de diagnóstico do aprendiz tem por objetivo identificar se o aprendiz possui habilidades e competências dentro do domínio específico. O modelo de habilidades e competências é estabelecido conforme orientações do $\mathrm{MEC}^{1}$. Tal modelo recomenda que se definam as competências esperadas e as habilidades correspondentes, que quando alcançadas, conduzam à obtenção das competências e as bases tecnológicas necessárias à aquisição das habilidades.

A Tabela da Figura 4.5 apresenta um exemplo de especificação de tais elementos, para uma unidade de aprendizagem sobre elaboração de casos de uso, para uma disciplina de Engenharia de Software de um curso de graduação. Proporciona uma visão geral dos resultados esperados com a aprendizagem, bem como dos recursos necessários (bases tecnológicas) para se obterem esses resultados.

\begin{tabular}{|c|c|c|}
\hline Competências & Habilidades & $\begin{array}{c}\text { Bases } \\
\text { Tecnológicas }\end{array}$ \\
\hline \multirow{3}{*}{$\begin{array}{lll}\text { Definir o papel de } \\
\text { casos de uso no } \\
\text { processo } & \text { de } \\
\text { software } & & \end{array}$} & $\begin{array}{l}\text { Especificar Requisitos de } \\
\text { SW }\end{array}$ & \multirow{3}{*}{$\begin{array}{l}\text { Requisitos } \\
\text { Casos de Uso } \\
\text { Processo de } \\
\text { Software }\end{array}$} \\
\hline & $\begin{array}{l}\text { Identificar a importância dos } \\
\text { casos de uso }\end{array}$ & \\
\hline & & \\
\hline
\end{tabular}

Figura 4.5. Modelo de Competências e Habilidades

Nesse processo, o modelo do aprendiz é construído com base em dois mecanismos de coleta de dados. O primeiro é por meio da aplicação de enquetes cujo objetivo é a identificação dos conceitos que o aprendiz já sabe. Cada questão possui um conjunto de conceitos relacionados, onde as respostas certas indicam que tais conceitos foram assimilados pelo aprendiz.

O segundo mecanismo de coleta de dados é realizado por meio dos registros de conteúdos visitados, assumindo que, se o aprendiz leu o conteúdo explicativo sobre um determinado conceito, visualizou exemplos e realizou exercícios corretamente, os respectivos conteúdos estão assimilados e representados no modelo do aprendiz.

A Figura 4.4 mostra o modelo CPN para o processo de diagnóstico do aprendiz para o primeiro mecanismo. $\mathrm{O}$ processo de diagnóstico testa se o aprendiz possui um conjunto de habilidades a fim de inferir suas competências.

\footnotetext{
${ }^{1}$ Pareceres CNE/CES n ${ }^{\circ} 776 / 1997$, CNE/CES n ${ }^{\circ} 436 / 2001$, CNE/CP n ${ }^{\circ}$ $29 / 2002$
} 


\section{Apresentação do Problema}

O critério de seleção do problema deve estar relacionado ao conhecimento representado no modelo do aprendiz. O lugar Problemas da Figura 4.5 guarda marcas do tipo $\mathrm{PB}$, cuja estrutura guarda o número e o enunciado do Problema. A Figura 4.5 mostra a CPN da etapa Apresentação do Problema.

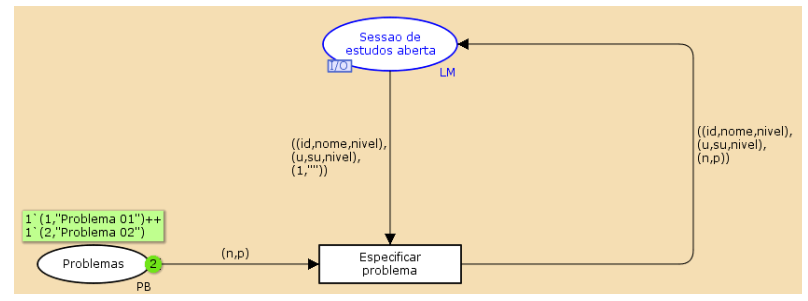

Figura 4.5. Modelo CPN da etapa Apresentação do Problema.

Após o disparo da transição Especificar Problema o estado Sessão de estudos aberta recebe uma marcação contendo a atualização dos dados do aprendiz e o problema apresentado. Esta especificação, neste modelo, é independente de regras.

\section{Assimilação}

A transição Assimilação é constantemente habilitada, exceto se não há marcações presentes no lugar Sessão de estudos aberta. Por limitação de espaço, alguns processos desta etapa são omissos. No entanto, a Figura 4.6 mostra as possibilidades que o aprendiz tem de interagir com o STI-PBL para acessar explicações de conceitos, visualização de exemplos e resolução de exercícios.

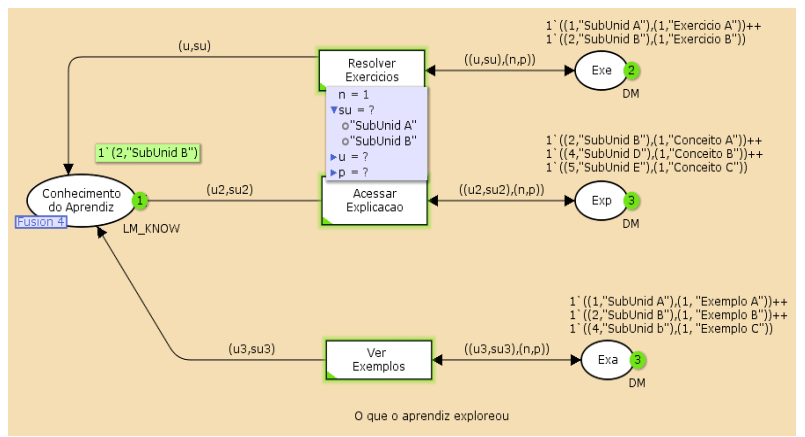

Figura 4.6. Submodelo CPN da etapa Assimilação

O lugar Conhecimento do Aprendiz da Figura 4.6 é um lugar de fusão que representa o conhecimento do aprendiz, obtido por meio da investigação dos assuntos de aprendizagem. A marca presente no lugar Conhecimento do Aprendiz significa que o aprendiz, identificado pelo código 2, possui o conhecimento da $\mathrm{Su}$ bunidade "B".

\section{Resolução de Problemas}

O processo de solução de problemas do STI-PBL é baseado no modelo Especialista caixa de vidro ilustrado na Figura 3.2.

A Figura 4.7 corresponde a uma CPN que representa dois processos de solução. Os requisitos para a execução de um passo no processo de solução do problema é representado por inscrições no arco de entrada das transições, as quais representam passos no processo de solução.

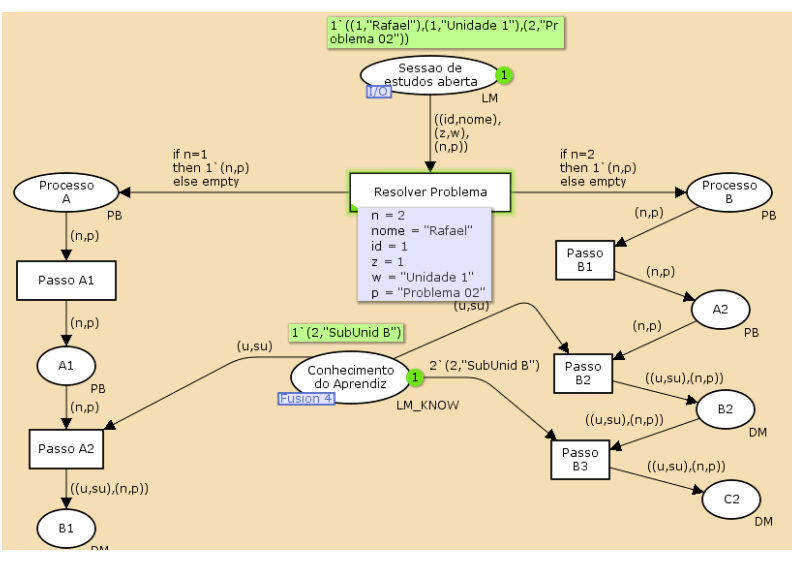

Figura 4.7. Submodelo CPN da etapa Resolução de Problemas

A ausência de conhecimento representado no lugar de entrada Conhecimento do Aprendiz impede que as transições Passo A2, Passo B2 e Passo B3 sejam habilitadas e, com isso, o aprendiz torna-se impedido de continuar no processo de solução escolhido. A Figura 4.7 ilustra a representação do processo de solução da fase Resolução do Problema.

Um dos objetivos desta pesquisa é identificar o momento mais apropriado para oferecer ajuda ou avaliação, durante o processo de solução, evitando que o aprendiz permaneça por muito tempo em um caminho errado, sem receber orientação.

Nota-se que o modelo CPN permite a avaliação dos passos intermediários no processo de solução, pois a ausência de marcas nos lugares de entrada de uma transição pode representar a ausência de requisitos para a solução do problema, propiciando ao tutor a visão de um momento apropriado para oferecer ajuda.

Esta percepção é possível pela análise das propriedades comportamentais da rede. Por exemplo, a ausência de marcas no lugar Conhecimento do Aprediz da Figura 4.7 indica que o aprendiz não assimilou os conceitos que são importantes para as estratégias de solução modeladas. Ele avançará até um determinado ponto, identificado na rede por uma transição denominada Passo $X_{i}$, mas não alcançará o estado objetivo.

O problema apresentado aparece na rede da Figura 4.7 intitulado Problema 02 e possui como pré-requisito a aplicação de um conceito, o qual é mostrado como marca 
presente no lugar Conhecimento do Aprendiz da forma 1`(2,"SubUnid B"). Esta marca é responsável por habilitar a transição Passo $B 2$ do processo $B$. Qualquer marca presente no lugar Conhecimento do Aprendiz torna a transição Passo A2 do processo A habilitada.

Em STI-PBL, os caminhos para a aprendizagem podem ser descritos em termos de seqüências finitas de disparo de regras (transições) e mudança de estados, onde cada estado representa um passo no processo de aprendizagem e solução de problemas.

\section{Verificação Formal}

A verificação formal consiste em submeter o modelo e as especificações a uma ferramenta. Essa ferramenta produz como resultado um valor verdade que indica se a especificação é satisfeita ou não no modelo. Em caso negativo, o verificador fornece uma sequiência de estados alcançáveis, chamada de contra-exemplo, demonstrando que a especificação não é válida no modelo [23]

$\mathrm{O}$ método aplicado para análise formal das CPN foi a árvore de alcançabilidade. $\mathrm{O}$ objetivo de aplicar esse método é analisar o estado da rede em um determinado momento, bem como a seqüência de disparos de transições que fazem com que o sistema alcance um estado objetivo.

A ferramenta CPN Tools simula os comportamentos da rede e retorna suas propriedades. Para as CPN da seção 4, foram encontradas as propriedades vivacidade, a qual significa que não existe um estado onde todas as transições são desabilitadas para o disparo, o que representa que o aprendiz sempre terá uma opção de interação com o sistema.

O relatório de espaço de estados informa que a rede foi avaliada parcialmente. Isto acontece porque a quantidade de estados encontrados no modelo é alta. O ideal, nesses casos, é construir redes menores, pois a análise da rede seria mais rápida e com menor risco de ambiguidades.

\section{Aspectos Funcionais Verificados}

Os aspectos aqui considerados correspondem a regras de negócio da aplicação. Essas regras normalmente são expressas de modo não formal. A simulação interativa na ferramenta CPN Tools permite ser controlada manual ou dinamicamente. Citam-se a seguir alguns aspectos funcionais verificados:

- sessão aberta de um aprendiz iniciando uma sessão de estudos;
- possibilidade de estabelecer cooperação entre aprendizes;

- como identificar evidências;

O detalhamento de cada um dos aspectos acima citados é apresentado a seguir.

\section{Sessão aberta de um aprendiz iniciando uma sessão de estudos}

A Figura 7.1 mostra o modelo CPN em seu nível mais alto, com dois aprendizes aptos para iniciar uma sessão de estudos. O lugar Aprendiz pronto possui uma marcação que representa dois aprendizes.

Para o modelo CPN da Figura 7.4, a marcação inicial indica que há dois aprendizes prontos para iniciar uma sessão de estudos e a transição Iniciar sessão de estudos está habilitada. Quando a transição Iniciar sessão de estudos é disparada, a marca correspondente ao aprendiz alterna de lugar e a transição Finalizar sessão torna-se habilitada.

A maioria das interações que o aprendiz pode fazer no STI-PBL é dependente do conhecimento representado no modelo do aprendiz e do problema apresentado. A transição Iniciar sessão de estudos da Figura 7.4 mostra que há dois aprendizes habilitados para iniciar uma sessão de estudos, permitindo a seleção manual ou dinamicamente.

\section{Possibilidade de estabelecer cooperação entre aprendizes}

Observa-se na Figura 4.7 que, a transição Passo B2 do processo $B$ não se tornou habilitada após a inclusão da marca (1, "SubUnid B") no lugar Conhecimento do aprendiz. Isso ocorreu porque a inscrição do arco (Conhecimento do Aprendiz X Passo B2) requer, exatamente, duas marcas do tipo LM_KNOW, que corresponde a (2, "SubUnid B").

Se for inserida na rede uma marca, ainda que de outro aprendiz, representando o conhecimento "SubUnid B", atenderá o requisito da inscrição do arco e a transição Passo B3 tornar-se-á habilitada.

\section{Como identificar evidências}

O lugar Conhecimento do Aprendiz guarda marcas do tipo LM_KNOW. Esse tipo representa o que o aprendiz sabe e o problema que ele está resolvendo.

Uma evidência no processo de solução significa um conhecimento representado no modelo do aprendiz que corresponde a um requisito de um problema. Os processos de investigação da fase Assimilação 
identificam o que o aprendiz sabe. Tais processos representam evidências observáveis, ou seja, quais dos requisitos do problema são conhecidos pelo aprendiz.

A identificação de evidências pode ser aplicada pelo modelo de adaptação em tomadas de decisão.

\section{Análise dos resultados}

As CPN podem ser comparadas a um grafo resolvedor de problemas e podem ajudar a estimar a probabilidade de um estado objetivo ser alcançado com base em evidências observáveis. Evidências observáveis são aspectos dinâmicos e podem variar, a partir das atualizações no modelo do aprendiz, tais como a visualização ou assimilação de exemplos, bem como explicações e exercícios resolvidos sobre as unidades de aprendizagem que estão relacionadas com o problema.

Para cada novo estado alcançado, o lugar é analisado e um conjunto de conceitos é esperado que esteja representado no modelo do aprendiz. Se estes conceitos não são representados, o modelo de adaptação poderá aplicar axiomas, heurísticas ou regras que auxiliará na tomada de decisão. É um modo de capacitar o tutor para identificar uma oportunidade para fazer inferência e adaptação.

Dos aspectos verificados, pode-se constatar que:

- o aprendiz tem acesso a todos os recursos da rede, logo, terá acesso a todos estados do STI-PBL.

- colocando dois aprendizes iniciando a sessão de estudos simultaneamente, observa-se que há pontos em que eles se encontram no mesmo estado, resolvendo o mesmo problema. Um pode cooperar com o outro na tarefa de solução. O modelo permite encontrar o melhor momento para recomendar cooperação.

\section{Trabalhos Relacionados}

Alguns trabalhos no campo de STI são baseados em Redes de Petri. Silva at al [24] apresentaram a modelagem da arquitetura do Modelo MATHEMA [25] baseada em Redes de Petri Coloridas. Para derivar o modelo por CPN, construíram cenários do funcionamento de um agente, diagramas de seqüência para simular a troca de mensagens, lógica temporal e verificação do modelo de modo integrado. Nesta pesquisa, optou-se por construir os cenários funcionais por meio de Diagramas de Casos de Uso e Diagramas de Estados de Interação.

LIN et al [26] preocuparam-se em aplicar Redes de Petri na especificação das seqüências de aprendizagem, como meio de adaptação para diferentes estratégias de ensino, de acordo com as habilidades individuais de cada aprendiz. Nessa abordagem, os assuntos de aprendizagem seguem uma ordem pré-estabelecida e, de acordo com a navegação do aprendiz pelo sistema, as unidades de interações disponíveis em cada lugar da rede tornam-se acessíveis ao aprendiz.

Mathtutor Shell [27] é um STI que tem interação adaptativa. Neste trabalho, Redes de Petri Hierárquicas foram usadas. O primeiro nível da rede representa o fluxo e suas camadas são conexas por um lugar de fusão, onde, na primeira rede é modelado o problema e seus prérequisitos, enquanto na segunda, para cada problema, todas as unidades de interação disponíveis. O que não é claro neste modelo é a representação das regras para a adaptação e como o conhecimento representado no modelo do aprendiz interfere no mecanismo de adaptação.

A metodologia aplicada na modelagem presentes em [25] e [27] foi parcialmente adotada neste trabalho.

\section{Conclusões}

Encontrar o passo no qual iniciou o processo de solução de problemas em STIs é um desafio que requer duas estruturas claramente definidas. A primeira é a definição e o detalhamento da estratégia pedagógica, pois é a partir desse detalhamento que se obtêm as unidades de interações entre o aprendiz e o STI, isto é, a definição das transições. A segunda é a modelagem de problemas em termos de pré-requisitos e a definição das transições.

$\mathrm{O}$ uso de CPN para modelagem da interação e adaptação mostra-se um potencial de uso por ser um método formal com representações matemáticas que propiciam uma formalização dos fluxos de processos dentro do ambiente de aprendizagem, identificação dos estados passíveis de atualização e consulta do Modelo do Aprendiz e de possíveis inferências do STI-PBL com o aprendiz.

Adicionalmente, permitem mostrar todas as interações relevantes e disponíveis para cada estado do sistema, dando visibilidade aos processos do aprendiz, suas ações e estados alcançáveis, além de permitir a verificação das propriedades do sistema por meio de uma ferramenta de simulação.

A especificação formal possibilitou que os experimentos, baseados no recorte da PBL, fossem analisados e verificados.

A principal dificuldade enfrentada para a construção das redes foi estabelecer a semântica para lugares e as marcações. Tal dificuldade foi facilitada pela concepção 
da visão geral do sistema por meio dos diagramas de casos de uso e dos Diagramas de Estados e Interação, os quais deram uma visão dos requisitos e funcionamento do STI-PBL.

A arquitetura clássica para STIs embora defina as responsabilidades de cada elemento da arquitetura, mostra-se insuficiente para separar o conhecimento do especialista com as estratégias de ensino e mecanismos de inferência. A arquitetura para STI-PBL mostrou uma clara separação de interesses, mostrando que o mecanismo de adaptação pode estar relacionado, mas independente da estrutura de dados que armazena o conhecimento.

A abordagem de modelagem por CPN apresenta como benefícios a possibilidade de especificar, verificar e simular as principais funcionalidades do sistema, a possibilidade de ter as Redes de Petri funcionando em paralelo com o STI, realizar simulações para buscar identificar novas possibilidades do que o sistema pode oferecer, tais como ajuda, orientação, verificação do funcionamento do modelo e análise de desempenho do sistema.

Dentre as principais contribuições, destaca-se a identificação do estado mais apropriado para oferecer ajuda ou cooperação, evitando que o aprendiz permaneça muito tempo em um caminho errado. A PBL foi a estratégia pedagógica escolhida porque é uma abordagem que tem sido muito discutida.

Como trabalho futuro, pretende-se modelar o processo de solução por Redes de Petri Coloridas e Estocásticas (ou Markovianas), com objetivo de aplicar algoritmos probabilísticos para inferir se o aprendiz alcançará a solução do problema, dado o conhecimento representado no modelo do aprendiz e a estratégia de solução modelada no plano de ação.

Considera-se o conhecimento representado no modelo do aprendiz uma base de evidências observáveis e o conhecimento necessário para a solução do problema representado no modelo especialista variáveis de consulta. Adicionalmente, a aplicação do modelo para monitoramento e avaliação durante o processo de solução de problemas e da aprendizagem.

\section{Referências}

[1] M.C.POLSON,J.J.RICHARDSON. Foundations of Intelligent Tutoring Systems. Hillsdale: Lawrence Erlbaum Associates, 1988.

[2] H.L. BURNS, C.G.CAPPS. Foundations of Intelligent Tutoring Systems: an introduction. In: POLSON, Martha C.; RICHARDSON, J. Jeffrey. Foundations of Intelligent Tutoring
Systems. Hillsdale: Lawrence Erlbaum Associates Publishers, p. 1-19. 1988.

[3] J.M.P.OLIVEIRA, Modelo de Referência para Sistemas Hipermídias Adaptativos Educacionais. Tese de Doutorado do Instituto Tecnológico de Aeronáutica, 2003.

[4] N. A. N. BERBEL A problematização e a aprendizagem baseada em Problemas: diferentes termos ou diferentes caminhos? Interface: Comunicação, Saúde e Educação, v.2, n.2, p. 139-154, 1998.

[5] D.H.JONASSEN. Using cognitive tools to represent problems. Journal of Research in Technology in Education, v.35, n.3, p.362-381, 2003.

[6] L.R.C. RIBEIRO. Aprendizagem baseada em problemas: uma experiência no ensino superior. São Carlos: EduFSCar, 2008.

[7] C. HSU, et al. Implementing a problem solving system for physics based on knowledge map and four steps problem solving strategies. In: INTERNATIONAL CONFERENCE ON ADVANCED LEARNING TECHNOLOGIES, Kazan, 2002. Proceedings... Kazan: IEEE, 2002.

[8] J.M.P. OLIVEIRA; et al. Processo de prototipação da interação em STI que utiliza a Aprendizagem Baseada em Problemas como proposta pedagógica. In: SIMPÓSIO BRASILEIRO DE INFORMÁTICA NA EDUCAÇÃO, 16., 2005, Juiz de Fora. Anais .... Juiz de Fora: SBC, 2005. p.394 - 404.

[9] J. M. P. OLIVEIRA; D.MAIA; D.GALANTE; E.J.V. SA; J.S.TEIXEIRA; C.T. A Proposal for Modeling Learner Interaction in Educational Adaptive Hypermedia Systems Driven by a Pedagogical Model. In: International Conference on Advanced Learning Technology, 2005, Koahsiung, Taiwan. Fifith IEEE ICALT Proceedings, 2005. p. 59-60.

[10] S. LABIDI.; J.S.FERREIRA. Technologyassisted instruction applied to cooperative learning. In: INTERNATIONAL CONFERENCE FRONTIERS IN EDUCATION, 1998, Tempe. Proceedings... Tempe: IEEE, 1998. 
[11] LABIDI, S.; FERREIRA, J. S. Agent-based architecture for cooperative intelligent tutoring system. In: INTERNATIONAL CONFERENCE ON THE DESIGN COOPERATIVE SYSTEMS, 3., 1998, Cannes. Proceedings... Sophia-Antipolis: INRIA, 1998b.

[12] F. BARGES. Innovation in engineering education: the Aalborg model. In: IBEROAMERICAN SUMMIT ON ENGINEERING EDUCATION, 1.,2003, São José dos Campos. Proceedings ... São José dos Campos: Fundação Valeparaibana de Ensino, 2003.

[13] J.R. SAVERY; T.M.DUFFY. Problem based learning: an instructional model and its constructivist framework. BRENT G. WILSON: Constructivist learning environments: case studies in instructional design, v.35, n.5, p. 135-148, 1995.

[14] E.S.RAMOS. Especificação e Verificação Formal de um Modelo de STI-PBL por Redes de Petri Coloridas. 2009. 121f. Tese de Mestrado - Instituto Tecnológico de Aeronáutica, São José dos Campos.

[15] L.M. KRISTENSEN; S.CHRISTENSEN; K. JENSEN. The practitioner's guide to coloured petri nets. International Journal on Software Transfer Technology, v.2, n.2. p. 98-132, 1998.

[16] R. ZURAWSKI, M. ZHOU. Petri Nets and industrial applications: a tutorial. IEEE Transactions on Industrial Eletronics, v. 41, n.6, p.567-583, 1994.

[17] J.L.PETERSON. Petri Net Theory and the modeling of systems. Englewood Cliffs: Prentice-Hall, 1981.

[18] A.V. RATZER, et al. CPN tools for editing, simulating, and analysing coloured petri nets. Berlin: Springer, 2003.

[19] A.S.JACINTO, J.M.P.OLIVEIRA. An ontologybased architecture for intelligent tutoring system. Scientia, v. 19, p. 25-35, 2008.

[20] R.S.WAZLAWICK. Análise e projeto de sistemas de informação orientados a objetos. Rio de Janeiro: Campus/Elsevier, 2004. 302p. v.1. instructional tasks: goals, strategies, and tactics for interactive leaning. [S.1.]: Adaptive Learning Environments, 1992. (NATO ASI Series v.F85)

[22] Coloured Petri Nets Group. CPN tools. University of Aarhus. Disponível em: http://wiki.daimi.au.dk/cpntools/cpntools.wiki. Acesso em 05 de dezembro de 2008.

[23] C.L.RODRIGUES, C.L. Verificação de modelos em redes de petri orientadas a objetos. 2004. Dissertação (Mestrado em Informática); Universidade Federal de Campina Grande, Campina Grande.

[24] L.D.SILVA; H.O.ALMEIDA; A.PERKUSICH. Modelling and analysis of a multi-agent intelligent tutoring system based on coloured petri nets. In: ACIS INTERNATIONAL CONFERENCE ON SOFTWARE ENGINEERING RESEARCH AND APPLICATIONS, San Francisco, 2003. Proceedings... San Francisco: SERA'03, p. 276-281, 2003.

[25] E. B. COSTA. Mathema: a learning environment based on a multi-agent architecture. In: Brazilian Symposium on Artificial Intelligence. 12.,1995, Campinas. Proceedings ... Campinas: EDITORA (Lecture Notes in Artificial Intelligence, v.991), 1995. p. 141-150, v.991).

[26] H.W.LIN.; et al. Applying petri nets to model SCORM learning sequence specification in collaborative learning. In: INTERNATIONAL CONFERENCE ON ADVANCED INFORMATION NETWORKING AND APPLICATIONS, 19., 2005, Taiwan. Proceedings ... Taiwan: IEEE, 2005.

[27] L.B.FRIGO, J. CARDOSO, G.BITTENCOURT. Adaptative interaction in intelligent tutoring systems. In: INTERNATIONAL WOKSHOP ACM CONFERENCE ON HYPERTEXT AND HYPERMEDIA, 16., Salzburg, 2005. Proceedings... Salzburg: ACM, 2005. 